\title{
Integrated design strategies for envelope skins: user-driven adaptive design solutions to improve sustainability and energy efficiency in social housing
}

\author{
E. Cattani ${ }^{1}$, A. Ferrante ${ }^{1,2}$ \& R. Gulli ${ }^{1}$ \\ ${ }^{I}$ Department of Architecture, Bologna University, Italy \\ ${ }^{2}$ Department of Applied Physics, University of Athens, Greece
}

\begin{abstract}
Upgrading the environmental and energy performance of existing buildings has taken in recent years a central role in the issues related to sustainability and energy saving. More than $75 \%$ of the energy consumed for heating and air-conditioning is essentially wasted due to inefficient insulation wrap, poor sealing of windows and poor performances of the plants' system. The identification of the most appropriate retrofitting options is a topic of outstanding importance given the potential costs and impacts involved. To ensure replicable results across the EU context, the research methodology presented in this paper is based on the assumption that the solution has to be found in a multi-disciplinary process applicable to almost all the possible scenarios. Standardized interventions are not the effective and sustainable solutions that we are looking for, pre-made receipts are generally addressed towards one main issue are partly effective and not efficient considering the life cycle of the building.

The aim is to combine technologies and design tools in one integrated process: 1) Offering an interesting payback time. 2) Increasing the energy performances of the envelope through variable solutions which increase the participation of the users. 3) Overcoming the existing socio-economic barriers

Keywords: energy efficiency, envelope performance, user driven solutions, building retrofitting, social housing.
\end{abstract}




\section{Social housing refurbishment: a socio-technical dilemma}

Two third of the buildings that make up the Italian real estate assets have been designed in total absence of specific regulations and with very few considerations on energy efficiency [1]. The social housing stock has been conceived everywhere in Europe according to the modernist idea of the minimal residential unit. This solution has been then applied - with few considerations on the context - all over Europe, generating social housing complexes that are now facing very similar problems: small dwellings, mono-functional districts, concentration of weak minorities and immigrants, low quality of public spaces, dull blocks in a dull environment [2]. On the basis of forecast scenarios the recovery will cover the $80 \%$ of the market in the coming ten years [3]. Given the size and the high number of buildings - eleven million only in Italy [4] - that can be grouped in this analysis, it is clear that a punctual and local strategy cannot be effective. Analyzing the social housing condition in Europe, it is important to point out that the original addresses of the buildings have drastically and rapidly changed in the past decades generating an important incompatibility between the building and its inhabitants. Those areas that once where the physical representation of the socialist utopia, are now turning into segregation ghettos, mainly occupied by immigrants and elderly people; buildings are no longer capable of answering to inhabitants' needs. An ethnographic and architectural revision is needed. It is important also to consider the impact of the potential intervention on the people living in the building who are not intentioned to move out from their apartments during the refurbishment period. This aspect represents an under estimated factor in the evaluation of the intervention's feasibility. The central role of the dwellers in the process becomes evident, the authorization for intervention needs to be unanimous otherwise the refurbishment proposal - even if highly innovative from a technological point of view - will never be approved. Thus the question upon the feasibility of the refurbishment project is nowadays strictly related to the users' agreement. The revision of the process here proposed, starts from a direct involvement of the inhabitants in the design phase, seeking for the individuation of those attractive nodes that could be used as incentives in the realization phase to foster the inhabitants' consensus. Parallel to this social connotation of the social housing problem, there are technical considerations that need to be made. Legislation on energy efficiency was implemented in Europe only after 1975, when social housing sprawl was almost at the end. A systematic program for the refurbishment of the entire social housing compound would result in substantial energy and comfort improvements. Through the incorporation of new elements, replacement of facade pieces or even a whole building façade removal [5] it is possible to reach a reduction up to $40-50 \%$ of the total consumption of the building [4]. The suggestion here presented is outlining a qualitative improvement of the façade components through tailored solution that could best answer to the requirements of the specific building, considering the climate conditions, the user utilization and the orientation of the building. This can be achieved with the utilization - since the beginning of the design project - of simulation programs like Energy Plus that are helping in investigating the differences and the effectiveness of a set of 
possible intervention, studying the variables that could be modified and customized according to the climatic and usage condition of the specific building considered. The EnergyPlus building systems simulation module, with a variable time step, calculates heating and cooling system and plant and electrical system response. This integrated solution provides more accurate space temperature prediction - crucial for system and plant sizing, occupant comfort and occupant health calculations [6]. It has been estimated that many of the problems of the building can be significantly improved through specific intervention directly on the architectural envelope as it is of strategic importance for a sustainable refurbishment that goes in the direction of decreasing the energy impact. Therefore the central challenge of contemporary practice in building refurbishment consists in finding an integrated answer for the two sides of this dilemma above illustrated. On one hand the process needs to take into account the inhabitants' point of view and meet their approval, seeking for an alternative to the anonymity of these areas through user-oriented solutions. On the other hand there is an urgent need for a technical revision of the envelope as complex system to improve the performances, reduce the costs and offer products that can adapt to the different façade typologies and configurations of the existing building. The final output will consist in a collection of façade components that offer a wide set of flexible and adaptable variants, allowing the transformation of the façade from a simple insulation layer, up to the entire re-design of the envelope including volumetric addition and shape transformation. The proposal is leading towards a new production system of the set of façade products; the main guiding software will govern a direct inter-action between inhabitants and planners: the ones will insert the input of their needs and wishes, the others will revise the output according to the performances and economic feasibility guaranteeing the coherency of the entire system.

\section{Urban potential and scenario}

In many countries of the EU, the political parties are already moving towards a more flexible framework for energy renewal interventions. The open discussion on the potential of increasing the volume of existing building is an important occasion to review the current legislative framework in favor of an indispensable liberalization of the normative. The urgency for densification policies that Europe is facing requires a direct engagement from both the Research and Development sectors and the Industrial sector. Therefore the upcoming European edition of the Solar Decathlon (June 2014, Versailles) has identified the five central topic of the competition considering the main issues related to sustainability in European cities: density, mobility, sobriety, innovation, and affordability. In one sentence the project has to be designed in its environment [7]. Many of the highly efficient prototype of homes presented have been designed to be directly integrated in the urban tissue as volumetric additions, rooftop extensions and/or infill between existing buildings. 


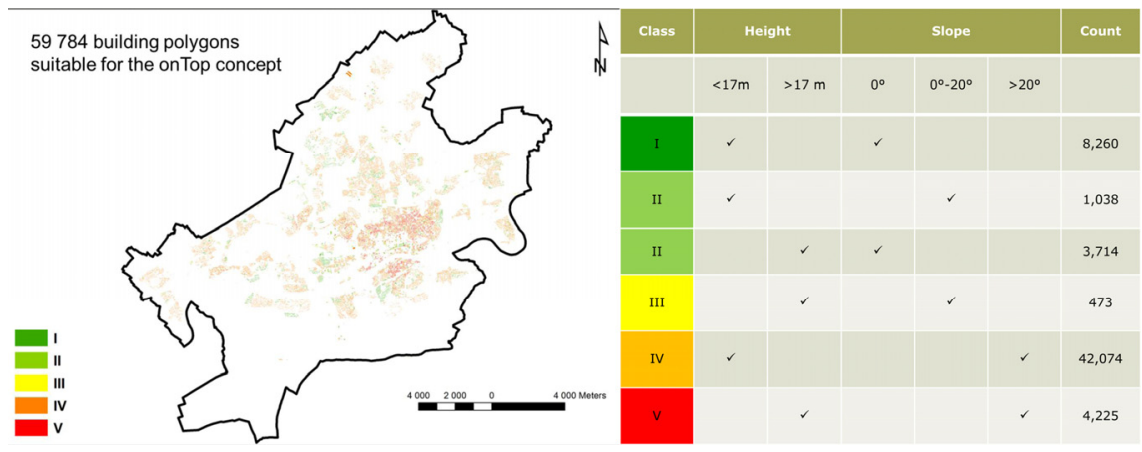

Figure 1: Results of the spatial analysis carried by the FH Frankfurt for the Solar Decathlon competition. On the left: classified building polygons suitable for the "OnTop" concept. On the right: count of building polygons according to their class [8].

The prototypes that will be built in Versailles as well as the preliminary studies and similar research experiences show the possibility of building this parasite architecture [9] through the utilization of light and prefabricated systems (wooden panels). This type of construction system will ensure the rapidity of realization, the reversibility of the intervention and will avoid structural interference with the static condition of the existing building that will 'host' the addition. The estimative research carried at the FH Frankfurt during the solar decathlon competition was aiming at a numeric evaluation of the potential of the OnTop strategy. The diagrams in Figure 1 shows the result of the spatial-urban analysis: around 60 thousand buildings in the city of Frankfurt am Main could be refurbished through on-top volumetric additions, showing the outstanding potential of the proposed action among buildings with urgent energy renovation needs. The highly replicable nature of this punctual densification, the important impact on the mobility and the social benefits that could outcome from the add-on densification strategy, demonstrate that this is more than just a proposal: it is the right path towards an effective renovation of our cities and a step forward in defining a new paradigm of sustainability.

\section{Volumetric addition, towards nZeb through a zero-cost strategy}

The proposed method to foster the Social Housing Refurbishment is made of three phases, each guided by a specific tool: the PfMatrix for the analysis, the OneToOne Milion Façade system for the parametric-controlled design of the façade components and the DesignITapp for the communication and inter-action with the inhabitants. The first two are digital software, studied to supports planners and stakeholders in the process, guiding a multi-variant parametric design while the latter is the result of the previous ones and groups 
together all the possibilities in one integrated collection of façade components that can be produced and industrialized file to fabric. The Path-finder Matrix (PfM) is the initial analysis tool developed by the research team to evaluate and compare the possible intervention scenarios. The matrix helps in identifying feasible instruments and actions to be applied within each different case study through a multi-disciplinary/multi-stake-holder comparison. Applying the matrix to the studied neighborhood, the stakeholders will obtain a complete overview of the possibilities towards a socio-technical intervention for the retrofitting [10]. The actions and instruments that are presented in the matrix are indeed a starting point and require to be implemented through the application of PfM in multiple scenarios: the scheme will be developed as an on-line open-source database of complete and immediate strategies in the social housing renovation sector. Starting from this overall and urban analysis the second step is focused to the building scale, seeking for instrument that can overcome the existing normative, financial and technical barriers in the process of energy retrofitting towards nZEB. So far, considering the research carried, the most effective approach has been identified in the volumetric addition metamorphosis of the envelope. The idea is based upon volumetric increase of the existing building through façade re-design and shows high financial potential in this context of economic slowdown. The costs of these interventions are not lower when compared with the standard energy retrofitting operations, but pilot EU experiences have already displayed that payback of larger scale interventions (considering roof extensions/elevations, construction of new units or densification) together with the direct benefit of real estate investors which gain space and money by possible add-ons, have a very positive effect on the technical and economic feasibility of the energy retrofitting interventions. Cost Benefit analysis performed in one of the case study showed that payback period of standard insulation resulting in 8-9 years with public incentives and 14 years without incentives. Considering the hypothetic investment of inhabitants in addons, the gains obtained by sales of the new flats are equal to $25.000 \mathrm{EUR} /$ unit, thus these gain would counterbalance both the standard energy retrofit (13.400 EUR per unit) and the cost of RES (pv panels) to set to zero the energy demand of the whole building. The elevated costs in energy retrofitting have driven some experimental design (i.e. SuRE-Fit, Reshape, etc.) as well as pilot experiences in Austria and The Netherlands to focus on the strategy of roof or side elevations/ extensions as additional volumes: the economic increment in terms of housing estate value is used to counterbalance the energy retrofitting costs. This incremental re-design of our cities is the right direction to answer to the increasing and worrisome land usage that Europe is facing. Only in Italy, 70 ha of land per day are used up for construction purposes [11], from 1990 up until 2005, Italy has lost 3,000,000 ha of agricultural land [4]. The energy renovation actions on the existing building could represent an important occasion to reduce the land usage, building on top of the existing tissue thus reducing the impact on the entire infrastructure net. It The volumetric add-ons on the roof of the buildings, as infill or façade extension represent therefore a powerful design strategy not only to foster the refurbishment process, gaining the economic surplus necessary to overcome the existing barriers but also an important occasion to sign a turning 
point in land and urban development, towards a new sustainable idea of planning and building smart cities.

\section{Parametric controlled façade components, One-To-One-Million Façade}

Looking at the design process, the proposal is based upon the idea of developing a collection of possible technological and formal solutions for the new envelope that should give the inhabitants the possibility to express their needs within a common order, respecting the guidelines of the project. This abacus (Figure 2) is thus based upon a set of parameters that could be changed and controlled by the planners, generating multi-variant options for the façade components. Since the 'letters' of this alphabet have been studied as a modular system and can be joined together in multiple combinations, the free 'discourse' that will be produced from inhabitants will have a positive result. The theoretical assumption is based on the possibility of developing multi-variant design for the facade refurbishment to form up to $1,000,000,000$ alternative versions. Moreover, the application of cnc machines and $3 \mathrm{~d}$ printers allows the design to go directly file-to-fabric, reducing the construction phase and the realization time. The façade component development within the prefabrication market has been influenced as well by the increase of customization techniques [12], the concept of 'mass customization' used by Stan Davis, refers to the industrial productive process of a property, according to a philosophy of maximization of the users satisfaction through the customization of the product performances. Considering the current dynamics of economic, financial, housing and energetic emergencies, the binomial 'mass customization/mass production' can nevertheless become an alternative key to the reading about the current and future tendencies in the field of the social housing [13]. The proposed shift in the design practice towards a mass-individualization and self-planning process, combined with the industrial tendency of developing mass customized products could turn the renovation practice in a common practice. One could argue that leaving to the inhabitants freedom in expressing their preferences brings no guarantee for the final composition of the façade; the authors reply that the formal outcome lies in the process and not in the final result, a project without content can result only in a mediocre design [14], the value of the building life cycle in a longer term retrofitting scenarios can in fact be increased only neglecting the use of universal and over-imposed solutions in favor of flexibility and adaptability in the design process.

Rehabilitate means acting on the interior of the housing, on the external factors and those collective with anoverall strategy [15]. Already several researches (ETH Zurich, 2011) have shown the potential of interactive systems to incorporate a high degree of adaptability within the renewal for office or retail spaces, investigating the possibility from the users to actively intervene on the functional distribution and layout of the building. Still very few has been said about how to incorporate the multiplicity of users needs and habits to the complex sector of social housing refurbishment, where rigidity is generally one of the main constrains. The procedure used by the research team to develop the design system foresees 
the utilization of Rhinoceros (MacNeel) as three-dimensional modeler and the Grasshopper plug-in to govern the parametrical variation of the geometrical dimensions. The Grasshopper plug-in is a graphical algorithm editor that allows designers with no formal scripting experience to quickly generate parametric forms [16]. The generic façade has been studied and mapped, building up a GH-definition that groups all the possible facades of a social housing building. Every dimension has been parameterized: the high of the floors, the window dimension, the balconies or loggias outline, the ratio between opaque and transparent surfaces etc. The planners can directly introduce the data that come from the survey on the existing building and immediately have a tridimensional model of the façade in Rhinoceros. The process continues with the isolation from the model of one module of the façade, correspondent to one apartment unit, this is the primary cell. The secondary cell is made up by the architectural span, generally repeated identically several times in the façade development while the entire building determines the tertiary cell. The division in cells allows the program to control the results of the multi-variant design at all the scales. In fact all the possible design solutions are then studied from the primary cell; according to the suggestions of the planners and the inhabitants a first set of options is produced. The variability of the different elements of the new façade are numerous and can be increased or changed according to the specific case study. A basic set of parameters that rule the existing façade has led to the definition of the main grid and order that is used as reference. This grid has then been transformed in the invariant structure that has a functional and skeleton use to support the variant elements of the façade collected in the abacus shown in Figure 2 together with the possible façade development. The coherency of the proposed solutions is verified through crossed checking with the secondary and tertiary cell, at all the possible scales. The dimension of the openings, the shading system, the depth of the volumetric addition, the materiality, the colors, the ratio between opaque and transparent surface can be changed and controlled in the GH-definition. A parametric transformation of these variables is specified and a numerical slider is customized to animate this transformation, providing immediate, high quality feedback from schematic design to its development.

In the third phase, the revision and discussion with the users is carried along, to engage inhabitants and owners in the selection and design process of the required solutions, giving life to creative, efficient but controlled self-expressed possibilities. Moreover it is important to underline that all the facade solution can be tested and simulated with Energyplus using the three dimensional model generated within Rhinocheros. This gives an exact and specific outcome of the performances that each of the chosen solution would guarantee and allows the planners to evaluate and compare the options with performance indicators related to the Energy Performance Index. Defining the main design principles and parameters according to their variability leads to the possibility of understanding and controlling a genotype that incorporates multiple design solutions. The single components of the façade (the phenotypes) can be interwoven, changed or transformed, still guaranteeing the coherence of the final composition as an open 


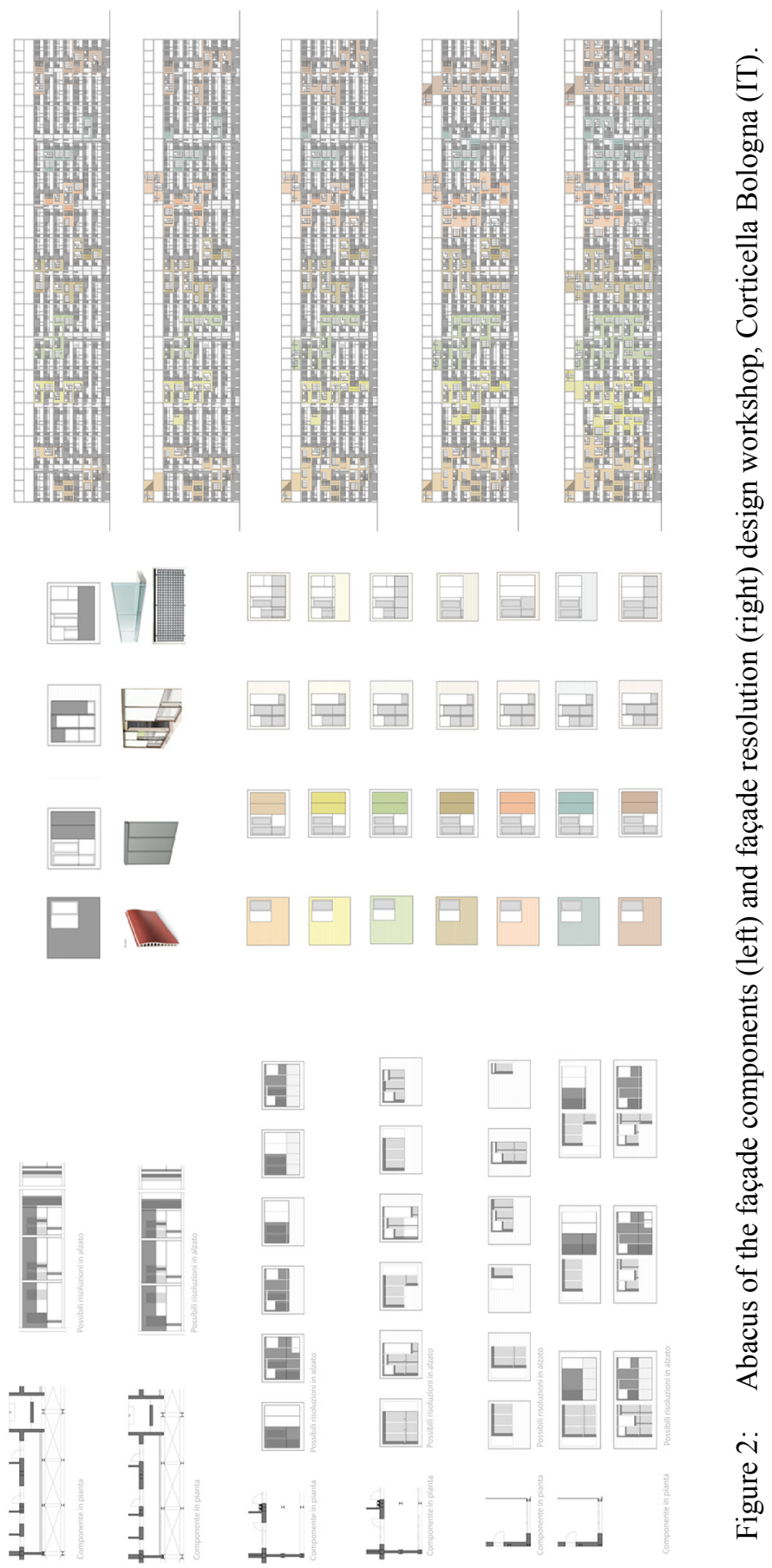


and in-fieri composition. The architecture is seen in the proposal as an infrastructure, as a set of supports [17] equipped with all the necessary plant and systems, able to host the different component of the façade as in-fill. The envelope is therefor made of adaptable elements that can transform and evolve also in time, according to the users new need and wishes in a metabolist perspective.

\section{The inter-active process of self-planning, DesingITapp}

The will of bringing the inhabitants back to the center of the design process has led to the definition of the third design tool, the DesignItapp. Design becomes a search of means for people to experiment different spatial possibilities, so they can evaluate, for example, where to place the openings as also the size of the rooms. As highlighted by Ghekiere (in [18]) the financial crisis has shown that a key element for the sustainability of any financial model for social housing consist of a system of intermediation, such as structures meant to create a link between the market and the providers. Interaction and feedback with the inhabitants in a trial and error matter, nowadays, is made possible and immediate in an inter-active manner through the sprawl of IT devices, smart phone and networks. The search for a method to communicate the possible design options to the inhabitant has led to the idea of developing an application for smart phone and tablets that could be downloaded directly by the users and 'speak' an easy and understandable language, translating the design process into a common practice. Dwellers and human being are different and more and more our multi-cultural society cannot fit with homogenous and standardized forms and schemes. More and more inhabitants tend to intervene on their dwellings given the inadequacy of the building in responding to environmental conditions. There is also an evident tendency in craving for ways to express their creativity and personal taste in turning a mere housing unit into a home. In this attempt it is possible to recognize the different cultural background and the very poor level of adaptability and flexibility allowed by the original layout of the buildings. Top-down actions usually ignore the dynamics of user's needs variability, and often result in standardized spaces. This corporate logic "is a consequence of an economic process in which it was "becoming uneconomic for the building industry to meet the specific needs of "users" for new products" [19] this means the end of diversity, and the progressive alienation of users. The existing barriers that are slowing down the process of renovation of our suburbs could be overcome by a proper transition from a merely technical approach towards an integrated sociooriented perspective. Therefore the starting point in developing the DesignITapp was the search for a definition of social sustainability of the renovation interventions based on an inter-active dialogue with the users, to reach a procedural revision of the construction practice and engage the inhabitants in the decision making phase. The idea was to communicate the $1,000,000$ possible façade transformations to the inhabitants and let them play with them, controlling the results but not imposing a choice, leaving the users free to decide what to do, to improve their apartments within the proposed options. Through the DesignITapp users will be asked to insert data regarding their habits and wishes to 
find out the existing conflicts between the buildings and their needs. A first set of façade transformation options will be displayed and the inhabitant will 'play' the role of the architect, experimenting the different solutions and changing them on the screen of their smart phones. A constant review will be provided by the DesignITapp, the basic and overall order set by the planners will be guiding the entire self-planning system. The different envelope options are presented with energy performance indicators, economic payback previsions, material-color variations and possible suppliers. The façade choice made by the user will then be evaluated through a grading system made of the mathematical average scores among three levels: grate of performance (EnScore), grade of price (EnEuro), grade of correspondence with the user need (EnYou). The scheme at the base of the parametric code ensures a cross checking with the choices of the other users in the building, at the three scales defined by the cells, as explained above. One solution is not fitting with all the others, there are only a certain number of possible combination paths for each solution and the warning error will be displayed in the decision making phase. It is not one design but a program that allows a series of design with a core invariant system and multiple outputs.

\section{Conclusions}

The actual need of considering a higher degree of variability and adaptability for the envelope to reach an effective sustainable system suggests a new approach, not only for the renewal practice but also for the design of new buildings. Indeed, the smart skin hypothesis proposes that if a building envelope possesses intelligence, it should be able to simultaneously take the needs of both users and environment into consideration [20]. The goal is to re-interpret the evolutionist theories to improve socio-technical environments in the specific contexts of energy retrofitting, collaborative learning, and architecture technology. The presented tool-kit provide planners with new multi-variant design tools, inhabitants can test the different options through an easy and direct application on their smart phones, evaluate them and chose the best option. Project managers are entitle of checking and guiding the entire process and guarantee an overall order that stands at the base of the process. The proposal consists in a digital revolution for the refurbishment practice to overcome the existing barriers and foster the entire renovation of social housing practice in Europe. This methodology has been tested in design workshops carried in Kanaleneiland post-war district Utrecht (NL), Corticella Bologna (IT), Peristeri Athens (GR) and Mousonstrasse Frankfurt (DE). The comparison between the results of the design researches show a very high degree of possible combinations: the design solutions have not been constrained to a simple prefabricated structure, indeed the need of developing modular and variable solutions has led to a wide set of possible architectural, technical and structural configurations. The different façade abacuses that have been produced for these case studies represent the base for the development of a new construction system for the façade production. The design becomes a game with no specific rules [13] between technicians and inhabitants. Ethnographic and cultural differences can be considered in the development of customized solutions 
to revision the envelope towards a more culturally responsive skin, keeping into account not only the performances aspects but also the cultural background of the inhabitants. The flexibility and inter-active character of the proposed system shows the high potential impact on the social housing renovation market: through the application of one process, the revision of millions of building would be possible, not through standardized intervention but with user-driven and tailored solution, different for each building and each user.

\section{References}

[1] Integrated European Project 2004, Brita in PuBs-Bringing Retrofit Innovation to Application in Public Buildings http://www.brita-in-pubs.eu/

[2] http://www.vanschagenarchitekten.com/

[3] Milardi, M., Retrofitting actions for a new housing quality, DASTEC, Housing for Europe, 2010

[4] Romano, A., 5 minuti di recupero, Teaspoon, Montaggio Claudio Esposito Sound design Procreate Lab

[5] Martinez, A., Noble, D., Schiler, M., Patterson, M., Retrofit Strategies for energy reduction in an office building in a mild climate, PLEA 2012 28th Conference, Opportunities, Limits \& Needs Towards an environmentally responsible architecture Lima, 2012

[6] Crawleya, B., Handb, J.W., Kummertc, M., Griffithd, B.T. Contrasting the capabilities of building energy performance simulation programs. Drury. Building and Environment 43 (2008) 661-673

[7] www.solardecathlon2014.fr Solar Decathlon Europe 2014

[8] Rikort, A., Markiewicz, M., Herrmann, M., Kreisel S., 3D Spatial analysis: Determining the buildings of Frankfurt of potential and suitability for the solar concept "OnTop", Fachhochschule Frankfurt am Main University of Applied Sciences www.geko-master.de, 2014

[9] Marini S., Architettura parassita, strategie di riciclaggio per la città, Architettura Ascoli Piceno, 2008 Quodlibet Studio

[10] Ferrante A., Cattani, E., Adaptability, Sustainability, Energy retrofitting: socio-oriented design strategies for zero-energy re-design of existing urban districts, ISUF CONFERENCE, 2012

[11] Il consumo di suolo in Italia, edizione 2014, rapporto ISPRA

[12] Pittini, A., Edilizia sociale nell'Unione Europea, CECODHAS Housing Europe Observatory, Brussels, Published in TECHNE Journal of Technology for Architecture and Environment, 04, 2012

[13] Di Sivo M., Angelucci F., Il mass customization process per l'Housing Sociale. Potenzialità, nodi critici e line di ricerca, ISSN online 2239-0243, Firenze University Press, 2011

[14] Friedman Y., Pro Domo, Actar Junta de Andalucia, Consejeria de Cultura, 2010

[15] Ginelli, E., Castiglioni, L., Perchè valorizzare e riqualificare il patrimonio di edilizia residenziale pubblico, Contemporary Architecture: Station Design for the Diffuse Loci of Travel, 2012 
[16] Lagios, K., Niemasz, J., Reinhart, C. F., Animated building performance simulation (ABPS) linking Rhinoceros/grasshopper with radiance/daysim, MA 02138 USA, proceedings of SimBuild, 2010 New York City

[17] Habraken, J., Supports: an Alternative to Mass Housing,Urban International Press, UK, Edited by Jonathan Teicher, 1999

[18] Pittini A., Edilizia sociale nell'Unione Europea, Techne 04, 2012

[19] Wanzel, J.G., 'Systems and society', Architectural Design, vol. 39, no. 10, London: Academy Editions, p. 565, 1969

[20] Chang C., Applying agent-based theory to adaptive architectural environment-example of smart skins, Smart Home Systems, Edited by Mahmoud A. Al-Qutayri, 2010 